\title{
An Overview on Extension and Limitations of Macroscopic Darcy's Law for a Single and Multi-Phase Fluid Flow through a Porous Medium
}

\author{
Suresh Kumar Govindarajan* \\ Professor, Reservoir Simulation Laboratory, Petroleum Engineering Programme, Department of Ocean \\ Engineering, Indian Institute of Technology-Madras, Chennai, India.
}

*Corresponding Author: Suresh Kumar Govindarajan, Professor, Reservoir Simulation Laboratory, Petroleum Engineering Programme, Department of Ocean Engineering, Indian Institute of Technology Madras, Chennai, India.

\begin{abstract}
Darcy's law is the fundamental equation that is used to describe the flow of fluids within a classical porous medium with a definite hydraulic conductivity and with a porosity varying between 0 and $100 \%$. This momentum conservation equation for describing isothermal single-phase fluid flow through porous media has been defined at the macroscopic-scale unlike the NavierStokes Equation (NSE) defined at the microscopic-scale, which is used to describe fluid flow through pipeswith 100\%porosity and infinite hydraulic conductivity. However, the extension of Darcy's law to describe the multi-phase fluid flow through a typical porous medium has severe limitations as an additional concept of an immiscible fluid-fluid interface along with its bounding surfaces. In addition, the concept of macroscopic capillary pressure associated with multi-phase fluid flow is traditionally related to the microscopic parameters as the mechanism of multi-phase fluid flow through a porous medium has not been conceived from the very fundamental conservation principles. The focus of the present paper is to revisit on the existing single-phase Darcy's law for both homogeneous and heterogeneous porous media followed by its extension to describe multi-phase fluid flow. Since, groundwater and petroleum communities are extensively using this conventional Darcy's law; and apply to almost all kinds of reservoirs including heterogeneous and fractured reservoirs; and also for compressible fluids under non-isothermal conditions in the absence of any modification or improvement. Hence, any forecast made based on using this simple Darcy's law requires careful understanding of this law and their serious limitations in depth. Understanding of such limitations on the extension of Darcy's law is highly required before finalizing the implementation of any subsurface fluid flow project. In this context, an attempt has been made by the author to deduce a list of serious limitations associated with the extended form of Darcy's law that takes into account the multi-phase fluid flow through both homogeneous and heterogeneous reservoirs. This exercise is expected to be of great help in the applied projects such as groundwater remediation, contaminant hydrogeology, enhanced oil recovery, $\mathrm{CO}_{2}$ sequestration, subsurface disposal of nuclear wastes, flow through fractured reservoirs, tight reservoirs, shale-gas reservoirs and CBM reservoirs, which require a critical understanding at the pore-scale fluid migration mechanisms with reference to that of macroscopic-scale Darcy's law.
\end{abstract}

Keywords: Darcy's law; multi-phase fluid flow; porous media; pore-scale; macroscopic-scale.

\section{INTRODUCTION}

The flow of fluids through porous media is a topic of immense practical interest in a great diversity of technical field such as groundwater recovery and management, contaminant transport analysis, subsurface disposal of nuclear wastes, enhanced geothermal energy system, oil and gas production, thermal/chemical/biological enhanced oil recovery, hydraulic fracturing, $\mathrm{CO}_{2}$ sequestration, combustion in inert porous matrix, flow through carbonate/fractured/shale-gas/CBM reservoirs, granular beds for the storage of solar energy, forced convection cooling and so on. Thus, characterizing fluid flow through a classical homogeneous porous medium; and subsequently, in a heterogeneous fractured reservoir has been the subject of immense interest in the context of groundwater aquifers (Suresh Kumar and Sekhar, 2005; Suresh Kumar et al., 2006; Sekar and Suresh Kumar, 2006; Sekhar et al., 2006; Suresh Kumar, 2008; Suresh Kumar et al., 2008; Suresh Kumar, 2009; Natarajan and Suresh Kumar, 2010; Natarajan and Suresh Kumar, 2010; Natarajan and Suresh Kumar 2011; Natarajan and Suresh Kumar, 2012a, 2012b; Renu and Suresh Kumar, 2012; 
Mohanasundaram et al., 2013; Berlin et al., 2013; Vasudevan et al., 2014a, 2014b; Suresh Kumar, 2014; Renu and Suresh Kumar, 2014; Natarajan and Suresh Kumar, 2014a, 2014b; Berlin et al., 2014a, 2014b; Natarajan and Suresh Kumar, 2015; Berlin et al., 2015; Nikhil and Suresh Kumar, 2015a, 2015b; Suresh Kumar and Rakesh, 2015; Suresh Kumar, 2015; Vasudevan et al., 2015; Berlin et al., 2015a, 2015b, 2015c; OmkarGaonkar et al., 2016a, 2016b; Renu and Suresh Kumar, 2016a, 2016b; Vasudevan et al., 2016a, 2016b, 2016c; Suresh Kumar, 2016; Nikhil and Suresh Kumar, 2016; Natarajan and Suresh Kumar, 2016; Vasudevan et al., 2017; Renu and Suresh Kumar, 2017a, 2017b; Samarth et al., 2017; Nikhil and Suresh Kumar, 2017; Mohanasundaram et al., 2017; Renu and Suresh Kumar, 2018a, 2018b; Suresh Kumar and Rakesh, 2018; Nitha et al., 2018; Natarajan and Suresh Kumar, 2018), petroleum reservoirs (Srinivasa Reddy and Suresh Kumar, 2014a, 2014b; Tusharsharma et al., 2014a, 2014b; Sivasankar and Suresh Kumar 2014; Tusharsharma et al., 2015a, 2015b, 2015c; Srinivasa Reddy and Suresh Kumar, 2015a, 2015b; Rockey Abhishek et al., 2015; Sivasankar et al., 2016; TapanKidambi and Suresh Kumar, 2016; RockeyAbhishesk et al., 2016; Sivasankar and Suresh Kumar, 2017a, 2017b; TapanKidambi et al., 2017; Suresh Kumar and Srinivasa Reddy, 2017; Patwardhan et al., 2017; Sivasankar and Suresh Kumar, 2018; Sivasankar and Suresh Kumar, 2019), geothermal reservoirs (Suresh Kumar and Ghassemi, 2005; Suresh Kumar and Ghassemi, 2006; Ghassemi and Suresh Kumar, 2007; Rakesh and Suresh Kumar, 2016; Bagalkot et al., 2018), shale gas reservoirs (Patwardhan et al., 2014; Samarth et al., 2015; Samarth et al., 2016), coal bed methane reservoirs and deep saline aquifers (Vivek and Suresh Kumar, 2016; Vivek et al., 2017). Since, flow through a classical homogeneous porous medium in itself has huge complexities, there are still a number of unresolved fundamental queries. For example, the fluid flow through a porous medium is assumed to follow a well-defined laminar flow regime, while in practice, it is actually the fluid flow through a complicated tortuous fluid flow paths associated with only hydraulically connected pore spaces in the absence of considering isolated or dead-end pore spaces. In addition, in the laminar regime, the fluid flow is supposed to be described solely by the viscous effects in the absence of any inertial or turbulent effects, while in reality, the fluid flow through a porous medium generally gets into a transition regime of Reynolds number, where the fluid flow is neither fully described by laminar nor by turbulent effects; but either by weak or strong inertial effects as well, depending on the applications of interest. Further, in describing fluid flow through both homogeneous and heterogeneous reservoirs, the geological formation of interest is assumed to be characterized by single-phase single-layered primary porosity, while in reality, it will also be characterized by a multi-phase multi-layered multiple-porosity systems. On top of it, for most of the applications, it is required to have the details at the microscopic scale, however, conceptually and mathematically simple macroscopic Darcy's law is widely used [Bradford and Leij, 1997; Diaz et al., 1987; Gray and Neill, 1976; Gray and Hassanizadeh, 1989; Hassanizadeh and Gray, 1990; Jerald and Salter, 1990; Kalaydjin, 1990; Liu et al., 2007; Muccino et al., 1998; Siddiqui et al., 2015; Teng and Zhao, 2000; Whitakar, 1986].Thus, there is a need to understand the fundamental concepts of the subsurface fluid flow at a scale lesser than Darcy's scale.For example, both groundwater and crude oil being the subsurface fluid flow, a litre of spilled crude oil in the subsurface environment can contaminate as much as one hundred thousand litres of groundwater; in addition, remediation of either onshore oil spill (contamination of groundwater by spilled crude oil) is not so easy as it will take several decades to address the same; and such field investigations require an understanding at the micro- or pore-scale processes and Darcy's law can only help us to address immediate concerns at a larger field scale; and any incorrect understanding of subsurface fluid flow processes will only land up with a marginal improvement in (enhanced) oil recovery factor. Since there is no mathematical model that can describe fluid flow through a porous medium at the microscopic-scale as on date, and since macroscopic Darcy's law is widely used till date by the oil and gas industries, it is necessary to understand the actual limitations associated with Darcy's law, when it is extended for various practical applications. It should be noted that Darcy listed his assumptions and limitations very clearly, while the same law was extended for various applications without actually realizing its limitations and assumptions. Thus, the focus of the current paper to bring out the possible list of assumptions and limitations associated with the Macroscopic Darcy's law, when it is used for various practical applications associated with the subsurface fluid flow.

\section{Homogeneous and HeTerogeneous Porous Media}

A porous medium can be conceptualized as a physical system composed of a solid part and a void space, where the solid part refers to the impermeable rock-matrix (or solid grains) and the void space refers to the permeable volumetric pore spaces through which the pore fluids such as water, oil and 
gas gets transported. There is a fundamental difference between a homogeneous and a heterogeneous porous medium. It can be noted that there is no separate storage space for pore fluids in a homogeneous and isotropic porous medium; and both the storage as well as transmission of the pore fluid(s) takes place at any given pore-space within the porous medium. However, in a heterogeneous fractured porous medium, the storage of the fluid(s) takes place within the low-permeable rockmatrix, while the transmission of the fluid(s) takes place through the high permeable fractures. Thus, the storage and transmission takes place in two different continua as against the single-continuum based homogeneous and isotropic classical porous medium.

Similarly, in a homogeneous and isotropic classical porous medium, the role of solid-phase resulting from the presence of solid-grains is generally avoided as it is assumed to be persistent and immobile in a homogeneous and isotropic porous medium, while in a heterogeneous and anisotropic porous medium, the restructuring of the pore geometry resulting from the reservoir compaction subsequentlyleads to the restructuring of solid-grains, unlike the assumption associated with a porous medium. And, in turn, the restructuring of the solid-grains leads to the changes in reservoir principal stresses; and in such cases, the fluid flow needs to be coupled with the geo-mechanical models in order to deduce the relation between the displacement and the pore pressure. Thus, in a homogeneous porous media, only the fluid is considered for the reservoir characterization and the solid-phase remains ignored, while in a heterogeneous and anisotropic porous media, it is required to consider the fluid flow along with geo-mechanical aspects.

It should be seriously noted that any heterogeneity or anisotropy associated with the microscopicscale or pore-scale should not be considered in the estimation of reservoir pressure or saturation; and only the macroscopic-level variations or fluctuations can be considered as the reservoir fluid dynamics is analysed based on the macroscopic-based (and not microscopic- or pore-based) Darcy's law. Even at the macroscopic-scale, the so called heterogeneity is not actually considered as the degree of heterogeneity depends on both the frequency as well as on the spatial distributionsof the variables of interest along with their inherent instability. Thus, it can be clearly seen that the heterogeneous parameters associated with the microscopic-scale should never be considered in the context of Darcy's law. On the other hand, the heterogeneity associated with the macroscopic-scale remains extremely challenging to capture those details at the larger field-scale. In essence, defining a reservoir either to be homogeneous or heterogeneous requires utmost care as what is required for the reservoir characterization is completely different from what is been actually carried out by introducing various assumptions.For example, in any reservoir characterization, the values of average reservoir porosity and the average reservoir permeability will be generally dealt with in the absence of their respective distribution, while in reality, the mean value of porosity should have been deduced, say, based on normal distribution, while the mean reservoir permeability should have been deduced, say, based on log-normal distribution. In addition, it is common to consider the permeability variations in the vertical directions only as the details on the depth wise depositional processes are relatively easier to secure, while the permeability variations in the horizontal direction is generally ignored despite the fact that the reservoir sedimentation very significantly over a typical reservoir length. In addition, in reality, the reservoir heterogeneity is described geologically as a function of reservoir structure, sedimentation and composition, while the same heterogeneity in practice is conceived as a function of reservoir porosity, permeability, fluid saturation and (net) pay thickness by a reservoir engineer, either from well-logs/core analysis or from pressure build-up data. Thus, the sample and the scale associated with the data collected from the aforementioned methods leads to nowhere in critically deciding the nature and degree of reservoir heterogeneity. It can be noted that the size of the core is infinitesimal with reference to the size of the reservoir, while the pressure build-up test provides the effective average (and not the simple average) value of the parameter over the drainage area of the well.In essence, there is no clear consensus on the systematic approach for investigating the nature and degree of heterogeneity associated with a reservoir in the context of reservoir performance evaluation.

In general, a porous medium can be conceptualized to be a homogeneous medium, when the parameters of interest have the same average value throughout the reservoir domain; and those values should have been deduced at the macroscopic-scale, where the minimum volume, over which the average value has been deduced, should merge with the Representative Elementary Volume (REV). 
On the other hand, if the mean value of the parameter keeps varying as a function of space, then, it is defined to be a heterogeneous reservoir. In addition, if the parameters of interest have the same average value, in a particular direction only, with differing values in the other directions (as against the same mean value throughout the reservoir domain), then, such a system is defined as an anisotropic medium. On the other hand, if the parameters of interest have the same average value in all the directions, then, such a system is defined to be an isotropic medium. From the above discussion, it is clear that in reality the actual concept of reservoir heterogeneity and anisotropy is not really taken into account towards reservoir characterization for various reasons; and using the same Darcy's law for almost all kinds of reservoirs deserves special attention with reference to the extension and limitations of Darcy's law.

\section{Single AND Multi-Phase Fluid Flow}

A phase can be defined as a portion of a system that is chemically homogeneous and which is separated from other such portions by a distinct fluid-fluid boundary. For a single-phase fluid flow, there is no fluid-fluid interface as the pore space is filled either with one fluid or with several fluids that are totally miscible with each other. For a multi-phase fluid flow, there is a definite fluid-fluid interface within the pore space as the pore fluids moving within it are totally immiscible with each other. Characterization of a single-phase fluid flow using Darcy's approach is straight-forward using groundwater or gases (gases are always totally miscible with each other), while the extension of Darcy's law to describe multi-phase fluid flow has serious limitations in the sense that there exists a concept of capillary pressure at the fluid-fluid interface, which has no relevance with Darcy's law. In addition, this concept of capillary pressure is associated with the pore-scale, while Darcy's law has nothing to do with the pore-scale scenario. In case of heterogeneous reservoirs, (a) the continuous interconnectivity of the pore fluids through the pore spaces; (b) the dimension of the pore space with reference to the mean free path length of the fluid molecule; (c) the presence of the adhesive forces at the fluid-solid interface; and (d) the presence of the cohesive forces at the fluid-fluid interfaces become very critical and sensitive; and in turn, the basis on which the treatment of a multi-phase fluid flow system in such heterogeneous reservoirsto be under continuum concept remains highly questionable. For describing multi-phase fluid flow, the capillary pressure; and in turn, the pressure in the wetting phase and non-wetting phase needs to be measured typically at the pore-scale (between 1 and 100 microns), while the macroscopic-scale based Darcy's law has nothing to do with such porescale processes.In fact, the reservoir fluid properties including density, viscosity and the miscibility are estimated at the molecular-scale, which is much lesser than the Darcy's macroscopic-scale. Further, the design of pore-geometry is expected to play a crucial role in the determination of the interfacial tension associated with the contact angle and the pore-pressure. Since, we do not have an idea about the individual void space configuration, it is not feasible to deduce the proper boundary conditions associated with each void space; and in turn, there is no way to describe fluid flow through a porous medium at the pore-scale as the pore path is so tortuous and complicated in nature.Further, there is no way one can measure capillary pressure in the field as on date; and in such a scenario, the extension of single-phase Darcy's law to describe the multi-phase fluid flow becomes highly questionable. But still, these parameters are assumed to follow a piecewise continuous function of space and time in order to attach it with the Darcy's macroscopic-scale. Thus, in order to avoid such pore-scale details, the Darcy-based macroscopic-law is widely used in order to describe the fluid flow through a porous medium, where only measurable statistical reservoir rock and fluid properties are required. In this context, understanding the extension of Darcy's law and its associated limitations would provide better insights towards an actual reservoir characterization.

\section{DARCY'S LAW AND ITS EXTENSION}

While describing Darcy's law, it can be shown that there exists a linear relationship between the Darcy flux and the hydraulic gradient. It should be noted that this relation has been deduced considering the fluid flow through a porous medium that involves the complete cross sectional area in the absence of any distinction between solid and fluid phases for describing the fluid flow. And, the mean fluid velocity, which is supposed to occur only through a hydraulically connected set of pores is mathematically deduced by dividing the Darcy flux with the effective reservoir porosity. It should be clearly noted at this point that Darcy established his empirical relation that correlates the volumetric fluid flux with the hydraulic gradient in the absence of reservoir porosity. Thus, the concept of mean 
fluid velocity was not discussed by Darcy. However, this mean fluid velocity has been experimentally found to be highly non-uniform; and characterized by fluid acceleration/deceleration along with significant changes in the direction of the fluid flow from its mean position. Further, the relation between Darcy flux and the hydraulic gradient becomes very sensitive at higher fluid velocities; and in such cases, the fluid flow could still remain laminar but the viscous forces that resist the fluid flow need not to be predominant; and the viscous forces can be acting along either with weak or strong inertial forces. However, it should be clearly noted that Darcy's law is valid only for the laminar fluid flow in the absence of any inertial forces. Thus, even, in a homogeneous and isotropic porous medium, it is relatively easier to establish whether the fluid flow is laminar or not, but, establishing the fluid flow to be either purely viscous forces or the viscous forces along either with weak or strong inertial forces is relatively difficult. And, the problem becomes still more complex for a heterogeneous porous medium. Thus, the deviation from Darcy's law associated with higher fluid velocities leads to a non-linear relation between volumetric fluid flow rate and the fluid velocity; and this relation remains sensitive for the creeping flow and the turbulent fluid flows. In essence, the linear relationship between hydraulic gradient and the fluid velocity gets broken down both at the low Reynolds number as well as at high Reynolds number. Thus, original Darcy's law does not include both porosity (rock property) as well as viscosity (fluid property), and for a porous medium involving higher fluid velocities, a possible non-linear relation can be found between Darcy flux and the hydraulic gradient with a given diameter of the solid-grains having a respective permeability; and this non-linear relation would remain independent of the reservoir porosity and fluid viscosity.

In analogy with Fourier's law of heat conduction, Darcy proposed an empirical relation that linearly connects the volumetric fluid flow rate $(\mathrm{Q})$ of an incompressible fluid with that of hydraulic gradient (I). The Darcy flux (or the Darcy velocity) refers to the volumetric fluid flow rate flowing through the entire cross sectional area of a porous medium as given in eqn. (1). This Darcy flux is proportional to the hydraulic gradient as given in eqn. (2). The parameter $\mathrm{C}$ represents the coefficient of proportionality as given in eqn. (3); and this constant is defined to be the hydraulic conductivity as given in eqn. (4).Eqn. (4) establishes the relationship between two measurable parameters namely the hydraulic gradient (I) and the Darcy velocity $\left(\mathrm{V}_{\text {Darcy }}\right)$ of the pore fluid. This empirical relation was formulated based on the limited ranges of volumetric fluid flow rates and the pressure drops with the assumption that there is no interaction between the fluid and solid phases within the porous media.

$$
\begin{aligned}
& V_{\text {Darcy }}=\frac{Q}{A}=q \\
& V_{\text {Darcy }} \propto I \\
& V_{\text {Darcy }}=C I \\
& V_{\text {Darcy }}=C I=K I \quad \text { for groundwater flow } \\
& Q=V_{\text {Darcy }} A=K I A=K\left(\frac{\Delta h}{L}\right) A
\end{aligned}
$$

In eqn. (5), $\mathrm{Q}$ represents the volumetric fluid flow rate $\left(\mathrm{m}^{3} /\right.$ day); $\mathrm{K}$ represents the hydraulic conductivity (m/day); I represents the hydraulic gradient (dimensionless); A represents the crosssectional area $\left(\mathrm{m}^{2}\right)$ normal to the direction of fluid flow; $\mathrm{V}_{\text {Darcy }}$ represents the Darcy velocity or Darcy flux ( $\mathrm{m} /$ day); and $\Delta \mathrm{h}$ represents the changes in hydraulic head over a distance L. Eqns. (1) - (5) corresponds to fluid flow through a porous medium associated with groundwater flow. If more than one miscible fluid is present as seen in coastal aquifers, with groundwater - sea-water interaction, then, the proportionality constant $(\mathrm{K})$ is directly proportional to the fluid density and inversely proportional to the fluid viscosity. Eqn. (5) can be for such cases can be rewritten as given in eqn. (6).

$Q=V_{\text {Darcy }} A=K I A=\left(\frac{k \rho g}{\mu}\right)\left(\frac{\Delta h}{L}\right) A$

In eqn. (6), $\mathrm{k}$ represents intrinsic permeability $\left(\mathrm{m}^{2}\right) ; \rho$ represents the fluid density $\left(\mathrm{kg} / \mathrm{m}^{3}\right) ; \mu$ represents the fluid viscosity $\left(\mathrm{N}-\mathrm{s} / \mathrm{m}^{2}\right)$; and $g$ represents the gravitational acceleration $\left(\mathrm{m} / \mathrm{s}^{2}\right)$. The hydraulic head (h) in eqn. (6) results from Bernoulli's equation with the assumption of insignificant kinetic head; and the expression for the hydraulic head is given in eqn. (7). 
$h=\left(\frac{P}{\rho g}\right)+z_{h}$

Eqn. (7) relates hydraulic head (h) with the fluid pressure. In eqn. (7), the first term on RHS represents the pressure head, while the second term represents the datum head, i.e., the height from a reference. The hydraulic head (h) and the fluid potential $(\Phi)$ are related by acceleration gravity $(\mathrm{g})$ as shown in eqn. (8). It can also be noted that the fluid always flows from high potential to low potential regions.

$\Phi=g h=\left(\frac{P}{\rho}\right)+g z_{h}$

Substituting eqn. (8) in eqn. (6) yields the Darcy flux as function of fluid potential and the expression for the same is given in eqn. (9).

$$
Q=V_{\text {Darcy }} A=K I A=\left(\frac{k \rho g}{\mu}\right)\left(\frac{\Delta h}{L}\right) A=\left(\frac{k \rho}{\mu}\right)\left(\frac{\Delta \Phi}{L}\right) A
$$

Going back to eqn. (5), there are no details about the pore-size or pore-geometry of the porous medium associated with the $\mathrm{K}$ value; and the value used in eqn. (5) represents the effective value representative of the entire porous medium.

Eqn. (5) can be modified into a differential form, when the limit of the quotient of the hydraulic head difference over the length of flow $\left(\frac{\Delta h}{L}\right)$ yields the negative of the hydraulic gradient as given in eqn. $(10)$.

$$
\operatorname{Lim}_{\Delta x \rightarrow 0} \frac{h(x+\Delta x)-h(x)}{\Delta x}=-\frac{d h}{d x}
$$

Substituting eqn. (10) in eqn. (5) yields eqn. (11).

$$
Q=V_{\text {Darcy }} A=K I A=-\left(\frac{k \rho g}{\mu}\right)\left(\frac{d h}{d x}\right) A
$$

In eqn. (11), the negative sign indicates that the direction of fluid flow is just opposite to the direction of the hydraulic gradient. In petroleum engineering, the concept of hydraulic head is not used; and instead, the pressure head is used as the oil and fields are associated with more than one pore fluid, unlike the single-phase groundwater flow. Eqn. (11) provided in terms of hydraulic gradient can be replaced with the pressure gradient as given in eqn. (12).

$$
Q=V_{\text {Darcy }} A=K I A=-\left(\frac{k \rho g}{\mu}\right)\left(\frac{d h}{d x}\right) A=-\left(\frac{k}{\mu}\right)\left(\frac{d p}{d x}\right) A
$$

Eqn. (12) can be expressed in terms of Darcy-flux (q) as expressed in eqn. (13).

$$
q=\frac{Q}{A}=V_{\text {Darcy }}=K I=-\left(\frac{k \rho g}{\mu}\right)\left(\frac{d h}{d x}\right)=-\left(\frac{k}{\mu}\right)\left(\frac{d p}{d x}\right)
$$

As can be seen from eqn. (13), Darcy's law can be seen as a momentum conservation equation, where the viscous force is being balanced by the pressure-gradient at the solid-grain - fluid boundary in the absence of inertial and body forces under steady-state conditions. In fact, eqn. (13) represents the momentum conservation equation for Stokes flow averaged over a Representative Elementary Volume (REV).Along the line of differential form, Darcy's law can also be expressed in terms of integral form as shown in eqn. (14). In eqn. (14), the intrinsic permeability (k) is directly proportional to the square of the mean grain size (or $\mathrm{d}_{10}$ or $\mathrm{d}_{50}$ or $\mathrm{d}_{\text {mean }}$ ). 


$$
\begin{aligned}
& q=\frac{Q}{A}=-\left(\frac{k}{\mu}\right)\left(\frac{d p}{d x}\right) ; \quad q \int_{0}^{L} d x=-\int_{p(0)}^{p(L)}\left(\frac{k}{\mu}\right) d p ; \quad q=-\left(\frac{k}{\mu L}\right)[p(L)-p(0)] \\
& q=-\left(\frac{k}{\mu L}\right)[p(L)-p(0)] ; \quad q=-\left(\frac{k}{\mu}\right)\left(\frac{\partial p}{\partial L}\right)
\end{aligned}
$$

Eqn. (15) is valid for a fluid flow in the horizontal direction; and eqn. (16) provides the expression for a tilted flow for the same case.

$$
q=-\left(\frac{k}{\mu}\right)\left(\frac{\partial p}{\partial L}-(\rho g) \frac{\partial Z}{\partial L}\right)
$$

Eqn. (16) represents the fluid flow against the gravity. However, when the fluid flow is towards the gravity, then, the sign in eqn. (16) gets reversed as shown in eqn. (17).

$$
q=-\left(\frac{k}{\mu}\right)\left(\frac{\partial p}{\partial L}+(\rho g) \frac{\partial Z}{\partial L}\right)
$$

The above equation works well for a steady-state, slow, laminar, one-dimensional single-phase fluid flow in a saturated homogeneous and isotropic, non-deformable porous media under isothermal conditions. But, in reality, we require that a subsurface fluid flow be described in multi-dimensions (2D or 3D); and for multi-phase, immiscible, compressible fluid flow in a non-homogeneous, anisotropic and deformable porous media under non-isothermal conditions.

In reality, the intrinsic permeability in the horizontal direction may vary as a result of large-scale tectonic stresses, fracturing and faulting, folding and any depositional processes and in such cases, the intrinsic permeability becomes anisotropic as against the assumed isotropic permeability; and in such cases, eqn. (13) gets modified into the following expressions in the three principal directions are given in eqns. (18) - (20).

$$
\begin{aligned}
& q_{x}=-\left(\frac{1}{\mu}\right)\left(k_{x x} \frac{\partial p}{\partial x}+k_{x y} \frac{\partial p}{\partial y}+k_{x z} \frac{\partial p}{\partial z}\right) \\
& q_{y}=-\left(\frac{1}{\mu}\right)\left(k_{y x} \frac{\partial p}{\partial x}+k_{y y} \frac{\partial p}{\partial y}+k_{y z} \frac{\partial p}{\partial z}\right) \\
& q_{z}=-\left(\frac{1}{\mu}\right)\left(k_{z x} \frac{\partial p}{\partial x}+k_{z y} \frac{\partial p}{\partial y}+k_{z z} \frac{\partial p}{\partial z}\right)
\end{aligned}
$$

Eqns. (18) - (20) represent the tensor form of intrinsic permeability. For a single-phase fluid flow, the term intrinsic permeability can in general be addressed as an absolute permeability. In the above equations, for example, $\mathrm{k}_{\mathrm{xy}}$ and $\mathrm{k}_{\mathrm{yx}}$ represent different fluid flow scenarios. $\mathrm{k}_{\mathrm{xy}}$ represents the fluid flow in $\mathrm{x}$-direction due to the pressure-gradient in $\mathrm{y}$-direction; and similarly, $\mathrm{k}_{\mathrm{yx}}$ represents the fluid flow in y-direction due to the pressure-gradient in x-direction and so on. Thus, eqns. (18) - (20) have been deduced to characterize an anisotropic and heterogeneous reservoir by replacing the constant intrinsic permeability with the spatially varying intrinsic permeability. In addition, if the porous medium is deformable and the single-phase fluid of interest is compressible (for example, gas), then, the Darcy flux (q) becomes a function of both velocity of water $\left(v_{x}^{\text {water }}\right)$ and velocity of solid $\left(v_{x}^{\text {solid }}\right)$ \{along x-direction as expressed in eqn. (21); and the hydraulic head (h) needs to be defined in terms of Hubbert's potential as given in eqns. (8) and (9).

$q_{x}=v_{x}^{\text {water }}-v_{x}^{\text {solid }}$

If there is more than one immiscible fluid in the pore spaces as seen in a petroleum reservoir, then, eqn. (13) slightly gets modified for the respective fluid phases as given in eqn. (22) - (25). 


$$
\begin{aligned}
& q_{i}^{\text {water }}=-\left(\frac{k_{i j}^{\text {water }}}{\mu_{\text {water }}}\right)\left(\frac{d p^{\text {water }}}{d x_{j}}\right) \\
& q_{i}^{\text {oil }}=-\left(\frac{k_{i j}^{\text {oil }}}{\mu_{\text {oil }}}\right)\left(\frac{d p^{\text {oil }}}{d x_{j}}\right) \\
& q_{i}^{\text {gas }}=-\left(\frac{k_{i j}^{\text {gas }}}{\mu_{\text {gas }}}\right)\left(\frac{d p^{\text {gas }}}{d x_{j}}\right)
\end{aligned}
$$

It can be noted that in eqns. (22) - (24), the permeability term represents the "effective permeability" as against the absolute permeability used in single-phase fluid flow; and this effective permeability is a strong function of the respective fluid saturation; and it represents the product of the absolute permeability and relative permeability as given in eqn. (25).

$k_{\text {effective }}=k_{\text {absolute }} k_{\text {relative }}$

In eqn. (25), the relative permeability represents the ratio of the effective permeability of a given fluid phase at a given saturation with reference to the absolute permeability. This relative permeability is a function of fluid saturation, wettability, imbibition-drainage history, pore size geometry and the distribution of the pore sizes; and thus the relative permeability directly influences the characteristics of the pore fluids; and in turn, influences the recovery efficiency of the respective reservoir fluids. However, there is no consensus on the unique relationship between the capillary pressure and saturation in the context of explaining the relative permeability as there is not an explicit consideration of specific interfacial area measured at the fluid-fluid immiscible interface. Thus, recent developments on multi-phase fluid dynamics have emphasized that the capillary pressure needs to be considered as a function of the interfacial tension (or "the change of interfacial free energy per unit change in interfacial area") rather than considering it as a simple function of wetting and non-wetting phase fluid pressures.

At higher fluid velocities than those dealt by Darcy, the relationship between the Darcy flux and the pressure gradient significantly deviated from linearity and the following expression considers the pressure gradient as a function of both first and second power of Darcy flux and it is expressed in eqn. (26).

$$
-\left(\frac{d P}{d L}\right)=\left(\frac{\mu}{k}\right)\left(\text { Darcy }_{-} f l u x\right)^{1}+(\beta \rho)\left(\text { Darcy_flux }^{2}\right.
$$

In eqn. (26), the $\beta$-factor is supposed to describe the morphology of the porous medium, which depends on many other parameters than porosity and permeability; and, as such there is no correlation that can be considered to be universal. However, it should be noted that at higher fluid velocities, the total pressure drop will result from kinetic energy loss $\left[2^{\text {nd }}\right.$ term on RHS of eqn. (26)] as well in addition to the viscous pressure drop $\left[1^{\text {st }}\right.$ term on RHS of eqn. (26)]. In general, the porous medium flow can be broadly characterized by three different regimes namely Darcy, Forchheimer and turbulent fluid flows based on the Reynolds Number (Re) as given in eqn. (27).

$\operatorname{Re}=\left(\frac{\rho q d_{\text {mean }}}{\mu}\right)$

In eqn. (27), q represents the Darcy flux (characteristic velocity of the porous medium), $d_{\text {mean }}$ represents the mean grain size of the particle (characteristic length of the porous medium $=$ pore size $\equiv$ mean grain size). In general, the transition from Darcy to Forchheimer flow occurs, when Re roughly exceeds unity, while the transition from Forchheimer to turbulent occurs, when Re roughly exceeds 100. In Darcy flow regime with $\operatorname{Re}<1$, the pressure drop is predominantly determined by the viscous pressure drop. The energy dissipation is generally maximum along the narrow pore-throats resulting from the solid-grain wall friction; otherwise, the streamlines are mostly steady and stable in this regime. With increasing Re from unity up to 100 , there will be weak inertial effect in addition to the viscous effect. This inertial effect causes the acceleration and deceleration of the fluid particle. In 
addition, the boundary layers develop significantly in this regime, while the flow still remains laminar. Re greater than 250 represent the laminar flow regime that is not steady, and the flow is characterized by weak oscillations, flow separation and the formation of eddies. Re greater than 400 represents the transient flow condition that is completely chaotic in nature as the streak lines breaks down. Thus, Re should be less than unity for the Darcy's law to be valid.

Apart from Reynolds number, Darcy number (Da) is also used, which is defined as the ratio between the intrinsic permeability of the reservoir to that of the square of the length-scale of the reservoir system as expressed in eqn. (28).

$$
D a=\frac{k}{L^{2}}
$$

In eqn. (28), $\mathrm{k}$ represents the intrinsic permeability, which can be expressed as a function of porosity as given in eqn. (29). Da increases with increasing mean grain size and pore size; and this number should be much less than unity for Darcy's law to be valid.

$$
k=\left(\frac{1}{180}\right)\left(d_{\text {mean }}^{2}\right)\left(\frac{\phi^{3}}{[1-\phi]^{2}}\right)
$$

Eqn. (26) can be further modified to include the third power of the Darcy flux to include much higher velocities as given in eqn. (30).

$$
-\left(\frac{d P}{d L}\right)=\left(\frac{\mu}{k}\right)\left(\text { Darcy }_{-} f l u x\right)^{1}+(\beta \rho)\left(\text { Darcy }_{-} f l u x\right)^{2}+\left(\gamma \rho^{2}\right)\left(\text { Darcy }_{-} f l u x\right)^{3}
$$

The steady-state Darcy's equation as given in eqn. (13) has been extended to its transient form by assuming the mean grain size of the particle to remain small so that the time-scale resulting from the division of mean particle size $\left(\mathrm{d}_{\text {mean }}\right)$ by the Darcy flux $\left(\mathrm{V}_{\text {Darcy }}\right)$ also remains small. However, it is to be noted that the time-scale over which the pressure change occurs is generally not small; and thus, the extension of Darcy's law to describe transient fluid flow condition also requires significant attention.

\section{LiMitations OF DARCY'S LAW}

Based on the earlier investigations, it was felt necessary by the author to have all the possible list of assumptions associated with Darcy's law at one place so that the scientists working on different applications can discuss their results, based on simplified Darcy's law with greater care; and it is expected that the details of the provided list of assumptions would critically help in deducing reservoir management decisions with utmost care.

1. Darcy's law does not describe the state of fluid flow within an individual pore.

2. Darcy's law does not take into account the actual curvilinear path of the fluid particle with continuously varying fluid velocity and acceleration scenario.

3. Darcy's law represents the nature of fluid flow at the macroscopic-scale as against the microscopic Navier-Stokes Equation (used for describing fluid flow through pipes).

4. Darcy's law does not take into account either weak or strong inertia, leaving aside the turbulent effect.

5. For Darcy's law to be valid, the porous medium is supposed to be isotropic, and, in addition, the porous medium should be averaged over the ensemble homogeneous.

6. Darcy's law is useful only for one-dimensional fluid flow, while for describing multi-dimensional fluid flow, the specific discharge or the Darcy flux needs to be treated as a vector quantity. In such cases, the Darcy flux becomes a vector point function and it may vary as a function of space and time in transient fluid flows. It can further be extended for an anisotropic system, where each principle coordinate will have respective Darcy flux with the condition that the maximum hydraulic-conductivity/intrinsic-permeability will be aligned perpendicular to the direction of 
minimum hydraulic-conductivity/intrinsic-permeability. In such anisotropic cases, the Darcy flux vector and the lines of fluid flow will not remain perpendicular to the surfaces with the same hydraulic head.

7. Darcy's law relates volumetric fluid flow per unit cross sectional area $\left(\frac{Q}{A}\right)$ to the hydraulic gradient $\left(\frac{d h}{d x}\right)$ and not to the pressure gradient.

8. Darcy's law relates the specific discharge (discharge per unit cross sectional area of the porous medium without introducing the concept of effective porosity; and in turn, the effective cross sectional area) to the gradient of the hydraulic head; and not the discharge as such.

9. Since, Darcy formulated the law for a steady-state fluid flow through a porous medium, the vector forces producing or driving the fluid motion can be equated to the opposing or retarding frictional forces; and the frictional forces opposing this fluid motion can subsequently be assumed to be proportional to the (i) volume of the pore-fluid $\left(\mathrm{V}_{\text {pore }}\right)$; (b) the Darcy flux $\left(\mathrm{V}_{\text {Darcy }}\right)$; and (c) the dynamic viscosity $(\mu)$. In other words, the various forces acting on the fluid must be in balance in the absence of fluid acceleration; and as a result, the subsequent vector sum of all these forces must be equal to zero at all points. Thus, it is to be noted that when estimating the fluid force normal to the cross sectional area (either on the upstream or downstream of the fluid movement), the fluid pressure should not only be multiplied by the cross sectional area but also by the effective porosity $\left(\phi_{\text {porosity }}\right)$. Similarly, the mass of the fluid that passes through a cross sectional area in a porous medium, either on the upstream side or downstream side, will not only be a function of fluid density $(\mu)$ and pore-volume $\left(\mathrm{V}_{\text {pore }}\right)$; but also will depend on the effective porosity ( $\left.\phi_{\text {porosity }}\right)$. This concept of (effective) porosity is absent in Darcy's law.

10. Since, Darcy conducted his experiments in a vertical saturated soil column, the gravitational component should have been considered explicitly as the vertical component of the gravitational force was also present along with the velocity vector resulted from the pressure gradient in the vertical direction of fluid flow. Thus, in this case with vertical column of experiments, two forces are acting in the direction of fluid flow; (i) the net pressure force; and (ii) the gravitational force that is acting (vertically downwards) in the direction of fluid flow. Since, both these forces have been oriented in the same direction of fluid flow, they can simply be combined by the vector addition. In addition, if the column is tilted from its vertical position, then, an explicit gravitational component parallel to the axis of the fluid flow will also contribute to the motion of the fluid in addition to the component resulting from pressure gradient; however, it should be noted that the magnitude of the total gravitational force vector acting in the vertical direction should not be considered as such. This is because the component of gravitational force normal to the direction of fluid flow axis or parallel to the direction of cross sectional area will not contribute to the fluid flow as this particular component will be balanced by the boundary (or wall) static forces; and also due to the fact that the gravitational force component perpendicular to the direction of fluid flow vector cannot provide any contribution to the dominant fluid velocity along the flow direction. Further, if the column is horizontal, then, the gravity vector cannot have a component normal to its original vertical direction as there is no horizontal component for gravity.

11. In describing Darcy's law, "the rate of change of pressure per unit distance" (or the pressure gradient, $\frac{d p}{d x}$ ) must be multiplied by "the distance over which this pressure change takes place" $(\Delta x)$, in order to obtain the actual pressure change along the direction of fluid flow. It should be clearly noted that the negative sign associated with Darcy's law results from the intrinsic negative quantity of "the rate of change of pressure per unit distance" acting along the direction of fluid flow as the fluid flows from higher potential to lower potential. In other words, the negative sign emphasizes the fact that the frictional retarding or opposing forces will be in opposite direction to that of the original direction of the fluid flow.

12. Darcy's law does not take into account the capillary and gravity effects. 
13. The term "Darcy velocity" associated with Darcy's law must be used with utmost care as Darcy's law relates the volumetric fluid flow per unit cross sectional area (called the Darcy flux) to the energy consumed per unit distance by the frictional resistance.

14. Darcy's original equation did not contain the pressure term as such; and it actually has the "hydraulic head" term directly associated with it. The hydraulic head gets converted into pressure term by applying the fundamental hydro-static principle $\left(h=\frac{p}{\rho g}\right)$.

15. Darcy's law assumes the porous medium to remain motion-less and non-deformable. The rate at which the solid gets deformed in a porous medium is assumed to be insignificant with that of the fluid deformation or fluid velocity. In other words, Darcy's law describes the movement of very slow flow of fluids in a porous medium with reference to the immobile or stationary solid-phase.

16. Darcy's empirical relation does not include the tensor form of the absolute permeability; and thus, the experimental/field validation of multi-dimensional permeability is beyond the scope of Darcy's law.

17. Darcy's law does not implicitly contain the parameters namely intrinsic permeability (rock property) and viscosity (fluid property); and the Darcy's original parameter namely, the hydraulic conductivity was later dissected into the above two components along with the gravitational acceleration.

18. Darcy's law ignores the fact the fluid flow actually takes place through preferential pathways (and not through all the pores); and also, ignoring those area of the pore spaces, which are stagnant.

19. Darcy's law is valid only for an incompressible fluid flow under non-isothermal conditions.

20. Darcy's law is associated only with the steady-state fluid flow conditions.

21. Darcy's law indicates that the fluid of interest in the porous medium is assumed to be under hydrostatic conditions. Thus, it is inherently assumed that any velocity variations caused within the fluid do not essentially dissipate any energy for a non-zero fluid viscosity' and it is also assumed that the movement of the fluid relative to the solid do not dissipate any energy for a nonzero intrinsic permeability. However, it should be clearly noted that such frictionless cases cannot be found in real reservoirs, associated with a typical porous medium.

22. The hydraulic conductivity term associated with Darcy's law should be used with care for the cases, where there are significant changes in mineral content of the fluid and/or in fluid temperature; and in such cases, it is better to use intrinsic permeability rather than hydraulic conductivity.

23. Darcy's law does not incorporate the effects of surface tension in the context of describing multiphase fluid flow through porous media, however, surface tension (or interfacial tension at the immiscible fluid-fluid interface) is the critical parameter that is used in the analysis of petroleum production / enhanced oil recovery.

24. Darcy's law assumes the fluid flow to be Newtonian on top of the fluid being incompressible; and hence, the resultant fluid flow pattern should remain independent of the fluid flow rate and the type of the fluid.

25. For non-Newtonian fluids, fluid viscosity becomes a function of applied shear rate; and in such cases, Darcy's law cannot be applied. Darcy did not accommodate the concept of "power law of fluids" in his empirical relation.

26. Viscoelastic fluids associated with drilling technology cannot be analysed using Darcy's law as the pore-fluid associated with Darcy's law should be a Newtonian fluid.

27. Darcy's law does not take into account the varying cross-sectional area from the production well (in case of radial flow); and such cases, it leads to a reduction in fluid velocity (away from the production well) for a constant flow rate. However, Darcy's law assumes a constant crosssectional area normal to the fluid flow direction. So, again, using Darcy's law for a radial coordinate has a serious concern. 
28. Darcy's equation does not actually get into the scale of Representative Elementary Volume (REV) as the size of the REV falls in between the micro-scale (discontinuous variable) and macro-scale (continuous variable); and it should be noted that the REV is much smaller than the continuum macro-scale. However, Darcy's law is not valid at the scale of REV, while it is valid only at the macroscopic-scale. Thus, the heterogeneities of a porous medium associated with the micro-scale has no relevance with Darcy's law. The situation becomes further complex for a heterogeneous reservoir. For such heterogeneous reservoirs, the permeability must be treated as a function of the spatial coordinates and the permeability becomes a tensor. Further, the fluid of interest may also be heterogeneous and in such cases, the fluid properties like density and viscosity needs to be treated as a variable. In essence, using average values of rock properties (like porosity and permeability) for the entire reservoir will not be helpful in capturing the actual reservoir heterogeneity using Darcy's law.

29. Darcy's law does not allow any point measurement; and the law requires the measurements to be taken only at the macroscopic-scale, i.e., in terms of the field measurements made as volumeaveraged quantities; and not as a point measurement. However, it can be noted that it is not practically feasible to deduce the volume-averaged velocity either by experimental investigations or by field means.

30. Darcy's law cannot be applied in the vicinity of either injection or production wells as the flow profile becomes curvilinear; and in turn, the fluid velocity has its components in both horizontal as well as in vertical directions. However, Darcy's law is applied only when the fluid flow within the reservoir is perfectly horizontal.

31. Darcy's law is valid only after a threshold pressure gradient resulting from frictional effects. Darcy's law fails beyond the minimum and maximum limits of Reynolds number. Experimental studies have shown the deviation of Darcy's law at Re as low as 0.1 and at as high as 70 . However, defining the critical Re remains very challenging.

32. The characteristic length associated with Re is difficult to deduce, and hence, the estimation of $\operatorname{Re}$ becomes challenging.

33. Darcy's law remains valid for a porous system with a constant pressure drop along a given direction in the absence of any inhomogeneous and time-dependent external forces.

34. Darcy's law assumes that the fluid flow is continuous through a series of hydraulically connected pore systems and ignores all isolated and dead-end pores.

35. Darcy's law is an empirical relation and not based on classical laws; and hence, the details of the critical porous medium parameters such as porosity, tortuosity, angularity, pore-size, porepacking, pore-sorting, specific surface area of the solid grains and grain size distribution are absent in the relation.

36. The extended macroscopic Darcy's law for describing multi-phase fluid flow needs to consider the pore-scale fluid-fluid interfacial boundaries associated with the wetting non-wetting phase fluid pressures. Hence, unlike a single-phase fluid flow, where the fluid pressureat any point in a porous system would remain the same, in a multi-phase fluid flow, the wetting and non-wetting phase pressures will not remain the same; and the balance between these two fluid-pressures pertain to the capillary pressure associated with the pore-scale.

37. The concept of capillary pressure associated with the multi-phase fluid flow requires to be defined at the macroscopic-scale (and not at the pore-scale) in line with macroscopic Darcy's law. However, this capillary pressure is a function of pore-scale parameters (and not the macroscopic scale parameters) namely interfacial tension and the radius of the hydraulic pore throat. At the macroscopic-scale, both wetting and non-wetting fluid phases are assumed to occupy a definite fraction of the pore fluid volume; and the interfacial area at the fluid-fluid interface becomes an aerial part of the 3-D pore volume at the pore-scale. Thus, it should be clearly noted that the curvature details of the fluid-fluid interfaces present at the pore-scale are not explicitly considered in the extended Darcy's law at the macroscopic-scale. In addition, in reality, it is not necessary that the shape of the fluid-fluid interface gets adjusted quickly with reference to the changes associated with the adjacent phases; and in turn, the capillary pressure may not be in equilibrium 
as the wetting and non-wetting phase fluid pressures change dynamically. In such circumstances, the assumption of equilibrium capillary pressure as a function of the difference between the wetting and non-wetting fluid phase fluid pressures needs a relook.

38. In describing multi-phase fluid flow, the wetting phase fluid remains disconnected and immobile from that of the mobile non-wetting phase fluid at irreducible water saturation. In such cases, the fluid pressure difference between the wetting and non-wetting phases may not reflect the real capillary pressure associated with the pore-scale. This capillary pressure associated with the nearly infinite capillary pressure would play a crucial role in deciding the macroscopic behaviour of the multi-phase fluid flow through a porous system.

39. The fundamental difference between single and multi-phase fluid flows arise from the fact that there exists a fluid-fluid interface for multi-phase fluid flows, which the macroscopic Darcy's equation does not directly consider this interface.

40. In case of multi-phase fluid flow, the fluid is not only driven by the pressure gradient and gravitational field but also by the presence of the adjacent immiscible fluid phase as well as by the bounding interfaces. The later component is generally ignored and the conventional multi-phase fluid flow equation does not accommodate the same.

41. The concept of capillary pressure associated with the multi-phase fluid flow is not only a function of fluid saturation, while it will also depend on the drainage-imbibition hysteresis; and in turn, the capillary pressure is no more a single valued function of the fluid saturation.

42. The existing multi-phase fluid flow equation assumes that the immiscible fluid-fluid interface has already reached the stable equilibrium shape; and in turn, the capillary pressure can be estimated as the difference between the wetting and non-wetting phase fluid pressures measured at either side of the fluid-fluid interface. However, in reality, there can be situations where the fluid-fluid interface does not remain at equilibrium; and it may get deformed dynamically. In such cases, the capillary pressure is found to be a function of the rate of change of wetting-phase saturation in the multi-phase fluid flow system. However, for an equilibrium capillary pressure, it becomes a function of both the saturation as well as the interfacial area per unit volume. Darcy's law does not consider all these microscopic details.

43. Fluid flow through a porous medium can be characterized by three major macroscopic quantities namely porosity, permeability and dispersivity (fluid particles gets dispersed as well), while Darcy's law accommodate only permeability in its empirical relation.

44. Darcy's law does not consider the cumulative effect on the drag of the fluid particles associated with the walls of the individual solid-grains.

45. Darcy's law gets violated at higher fluid velocities, where the reservoir permeability varies as a function of fluid velocity for a constant fluid viscosity.

46. Darcy's law gets violated at higher fluid velocities as the proportional increase in the Darcy flux with respect to the applied pressure gradient is actually lesser.

47. Even the macroscopic Darcy's law does not define the boundary conditions at the solid walls precisely; and in turn, it is difficult to model this problem mathematically to remain to be wellposed.

48. Darcy's law neither bother about the pore space of a porous medium explicitly nor the volume fraction of the pore space associated with the control volume.

49. The permeability term present in Darcy's equation does not vary as a function of scale.

50. Darcy's law does not describe that the permeability associated with it should follow the lognormal distribution.

51. The characteristic length associated with the Darcy's law pertains to the pore size of the porous medium, whose dimensions do not allow the fluid flow to be considered as a simple onedimensional flow.

52. The characteristic velocity associated with the Darcy's law does not reach its maximum velocity along the centre of the any given pore channel. 
53. Darcy's law does not accommodate the fluid flow at very low pressures; and hence, this law will not be able to take into account the slippage effect or Klinkenberg effect, where the intrinsic permeability becomes a function of pressure.

54. Darcy's law does not take into account the sensitive pore-scale detail that considers the shear stress at the entrance and exit of a pore and the normal stress at the wall of the solid grains; and this aspect becomes very sensitive in tight gas reservoir.

55. Darcy based macroscopic flow behave significantly different from the pore-scale based interstitial flow as the viscous stresses associated with a porous medium, not only act on the pore fluids, but rather, on the entire volumetric porous medium (that consists of both solid and liquid phases); and in turn, the resultant viscous force should not only be associated with the pore-fluid force but as the complete body force of the entire porous system at the macroscopic-scale. Thus, the flow may become rotational (and not irrotational as assumed in Darcy's law) at the pore-scale, while it is simply conceptualized to be a potential flow at the Darcy's macroscopic-scale.

56. The intrinsic permeability associated with Darcy's law is a function of square of mean grain size, while it is to be noted that the kinetic effect associated with the geometry of the "void" at the pore-scale would provide better insights than considering only the mean size of the "solid" (grain) in the permeability determination.

57. Darcy's law describes single-phase fluid flow, where the estimation of resistive force is a simple function of Darcy flux, the pressure gradient and the fluid viscosity in the absence of gravity effects. However, in reality, the resistive force will be function of pore size \& shape, pore-size distribution and the connectivity of theses pore spaces; and thus, in reality the resistive force will be varying as a complex function of space. In addition, when the fluid flow becomes multi-phase, then, the estimation of resistive force will become still more complex as it will depend on the degree of the contact of the multiple fluids with each other; the degree of contact of each fluid with the solid; on the saturation of the individual fluid within the pore space; and on the relative attraction of each fluid by the solid. In addition, the resistive force will differ for the porous medium that undergoes an imbibition process (wetting of the solid-grains) from that of the porous medium that undergoes a drainage process (drying of the solid-grains).

58. Darcy's single-phase fluid flow model was extended to describe the multi-phase fluid flow, where both the wetting-phase and non-wetting-phase relative permeability is generally assumed to be function of wetting-phase saturation only; and this value can vary between zero at the lowest wetting-phase saturation and unity for the $100 \%$ wetting-phase saturation (single-phase fluid flow). Thus, it should be noted that the summation of the wetting and non-wetting phase saturations will lead to unity, while the summation of the wetting and non-wetting phase relative permeabilities will always remain less than unity; and it subsequently indicates that the multiphase fluid flow through a porous medium is associated with the reduced over-all permeability of the porous system in comparison with that of its respective single-phase fluid flow system.

59. The extension of Darcy's law in describing multi-phase fluid flow assumes the net fluid forces in terms of pressure gradient and gravity forces associated with a particular fluid phase only (either wetting or non-wetting); and it does not include the forces resulting from the interactions between the solid-phase and the different fluid phases (wetting and non-wetting) concerned. For example, the very concept of wettability, which describes the preferential attachment of the solid phase by one of the fluid phases (interaction between solid and fluid phases; and it results in the attraction of the wetting fluid by the solid phase; and the solid holds the wetting fluid against the gravitational forces) is primarily missing in the conventionally used empirical relation for describing multi-phase fluid flow.

60. The single-phase Darcy's law assumes that the single-phase fluid moves in between the solid grains; and the interface between the solid and fluid phases is immobile, while in the case of multi-phase fluid flow, one of the immiscible fluids move in between solid and the other immiscible fluid. Thus, the interface between the immiscible fluids within the pore space is mobile unlike the stationary interface between the solid and fluid phases associated with the single-phase fluid flow. It is to be noted that out of these two immiscible fluids, the non-wetting 
phase fluid is driven by the force imbalance between the pressure gradient and gravitational field associated with this non-wetting phase, while there can be the mobility of the wetting-phase fluid resulting from the drag of the non-wetting phase fluid; and not from the force imbalance between the pressure gradient and gravitational field associated with the wetting phase. In other words, the imbalance or deviation resulting from the hydrostatic pressure distribution in non-wetting phase could cause flow of the wetting phase. And, this dragging force is absent in the conventional multi-phase fluid flow equations. Also, the treatment of the momentum transfer between the miscible and the immiscible fluid interfaces within the pore spaces require utmost care as the momentum transfer associated with the fluid-fluid interface is not the same as that of the momentum transfer associated with the solid-fluid interface.

61. The conventional equation used to describe the multi-phase fluid flow, based on the extension of Darcy's law does not accommodate the resistance resulting from the flow of both wetting and non-wetting fluid phases by the solid phase apart from the resistance resulting from the changes in fluid velocity between the two immiscible viscous fluid phases. Thus, the existing multi-phase fluid flow equations dictate the intensity of relative permeability to be a function only of fluid saturation, while in reality, it will also depend on whether which process (imbibition or drainage) has actually taken place.

62. The conventional equation used to describe the multi-phase fluid flow, based on the extension of Darcy's law use the concept of relative permeability for wetting and non-wetting phases. However, this relative permeability does not explicitly consider the rheology of the fluid-fluid interface; and the intensity of viscous coupling at the fluid-fluid immiscible interface depends on the interfacial shear viscosity. However, this effect is not given importance under general circumstances with the assumption that the hydrodynamic interaction between wetting and nonwetting fluid phases remain to be insignificant under dominant capillary forces at the pore-scale with reference to the viscous forces. Thus, the existing equations for multi-phase fluid flow is essentially described in terms of "hysteresis" as a function of the wetting and non-wetting phase fluid pressures; the relative permeability of the wetting and non-wetting phases; and the saturation of the wetting and non-wetting phases. This hysteresis results from the differences in wetting and non-wetting phase fluid saturations, dictated by the migration of the immiscible fluid-fluid interface.

63. The existing multi-phase fluid flow equations provide only one value of relative permeability for a given fluid saturation. However, in reality, there can be more than one value of relative permeability for a given fluid saturation, when the actual fluid saturation within the pore space is divided into three different components namely (i) a flowing fraction of the fluid; (ii) a nonflowing or immobile fraction connected to the flowing or mobile fraction of the fluid; and (iii) an isolated fraction disconnected from the flowing or mobile fraction of the fluid. Thus, there cannot be an universal unique relation for relative permeability as a function of fluid saturation only in describing multi-phase fluid flow dynamics, while the concept of relative permeability needs to be improved that will account for the contributions from the immobile and isolated fluid fractions within the pore spaces, apart from the details on mobile fluid fractions.

\section{CONCLUSiON}

The extended Darcy's law to describe the multi-phase fluid flow at the macroscopic-scale considers the reservoir geometric properties such as porosity, saturation and fluid-fluid interfacial area per unit volume, while these geometric properties do not exist at the pore-scale; and in turn, there is a clear disconnect between the existing macroscopic-based extended Darcy's law and the actual multi-phase fluid flow mechanisms happening at the pore-scale; and requires that modified or improved mathematical models need to be developed to formulate the mass, momentum and energy conservation equations at the pore-scale associated with the multi-phase fluid flow including an explicit interface equation. It is also observed that as long as the fluid-fluid interface sustains stress; and contributes to the dynamics of multi-phase fluid flow, there should an explicit equation for the interface despite its massless property.

In addition, it should be clearly noted that the extended Darcy's law pertains to the macroscopic-scale; and in such cases, validation of macroscopic-scale modelling results using pore-scale parameters such 
as contact angle and hydraulic pore-throat radius have wrong implications as the integration of these pore-scale parameters do not lead to extensive variables.

Since Darcy's law is widely used in practical applications such as remediation of groundwater, oil and gas extraction including enhanced oil recovery, thermal energy recovery from geothermal reservoirs, subsurface disposal of nuclear wastes, $\mathrm{CO}_{2}$ sequestration, flow through fractured reservoirs, shalegas/tight reservoirs and CBM reservoirs, there is a dire need that a mathematical model needs to be developed that can describe both the single and multi-phase fluid flow through a porous medium at the microscopic-scale so that the vital concepts such as pore-scale displacement efficiency used in Enhanced Oil Recovery (EOR); nanoparticle based chemical EOR; nanoparticle based groundwater remediation can be understood with greater significance; and in turn; it will help to improve the reservoir/aquifer efficiency. Care should be taken that different parameters become sensitive and dominant at various scales including nano-scale, molecular-scale, pore-scale, microscopic-scale, macroscopic-scale, laboratory-scale, pilot-scale and field-scale, while formulating the required mathematical model. Thus, ensuring connectivity between the mathematical models formulated at different scales will be of prime importance in the absence of deducing the macroscopic quantities such as pressure and temperature as the average over the microscopic-scale quantity. In fact, an exclusive mathematical model is required that will address mass, momentum and energy conservations within the wetting and non-wetting fluid phases as well as at the fluid-fluid interface. The associated fluid driving mechanisms at different scales need to be deduced with greater care as it is not always the pressure gradient (and the gravitational field) that drives the multi-phase fluid flow, while, it can be the chemical potential as well that drives the multi-phase fluid flow at the pore-scale. The assumption of fluid drive by the pressure gradient works well for the isothermal reservoir system; and also for the cases, where the interaction between wetting and non-wetting phases with its boundary remains insignificant. On the other hand, for a reservoir system with non-isothermal system; and also for the cases, where the interaction between the wetting and non-wetting fluid phases with its boundaries are quite sensitive, then, the fluid drive by the chemical potential will also play a crucial role in addition to the fluid drive by the pressure gradient. Further, the local interaction between the wetting and non-wetting phases becomes crucial, when the saturation of the wetting phase remains lower; and when this wetting phase is present in the form of thin films within the pores, distributed throughout the porous system. The storage and transport of mass, momentum and energy through the fluid-fluid interface needs to be formulated in detail at the pore-scale. And finally, the mechanism of capillary pressure at the pore-scale should be formulated as a function of pore-scale drainageimbibition hysteresis parameters as well in addition to the fluid phase pressures and fluid saturation.

\section{REFERENCES}

[1] Bradford S A., and F J Leij (1997). "Estimating interfacial areas for multi-fluid soil systems. Journal of Contaminant Hydrogeology, vol. 27, pp. 83-105.

[2] Bagalkot N., A. Zare., and G. Suresh Kumar. (2018). "Influence of Fracture Heterogeneity Using Linear Congruential Generator (LCG) on the Thermal Front Propagation in a Single Geothermal Fracture-Rock Matrix System". Energies (MDPI Publications), vol. 11(4), 916, pp. 1-13.

[3] Berlin, M., G. Suresh Kumar., and I. M. Nambi. (2013). "Numerical Modeling on the Fate and Transport of Nitrate in an Unsaturated System under Non-Isothermal Conditions". European Journal of Environmental and Civil Engineering (Taylor \& Francis), vol. 17, No: 5, pp. 350-373.

[4] Berlin, M., G. Suresh Kumar., and I M Nambi. (2014a). "Numerical Modeling on the Effect of Dissolved Oxygen on Nitrogen Transformation and Transport in an Unsaturated Porous System". Environmental Modeling\& Assessment (Springer Publications), vol. 19(4), pp. 283-299.

[5] Berlin, M., G. Suresh Kumar., and I. M. Nambi. (2014b). "Numerical Modeling on Transport of Nitrogen from Wastewater and Fertilizer Applied on Paddy Fields". Ecological Modeling (Elsevier Publications), vol. 278, pp. 85-99.

[6] Berlin, M., G. Suresh Kumar., and I. M. Nambi. (2015a). "Numerical Modeling of Biological Clogging on Transport of Nitrate in an Unsaturated Porous Media". Environmental Earth Sciences (Springer Publications), vol. 73(7), pp. 3285-3298.

[7] Berlin, M., M. Vasudevan., G. Suresh Kumar., and I. M. Nambi. (2015b). "Numerical Modeling on Fate and Transport of Petroleum Hydrocarbons in an Unsaturated Sub-Surface System for Varying Source Scenario". Journal of Earth System Science (Springer Publications), vol. 124(3), pp. 655-674.

[8] Berlin, M., I. M. Nambi., and G. Suresh Kumar (2015c). "Experimental and Numerical Investigations on Nitrogen Species Transport in Unsaturated Soil during Various Irrigation Patterns". Sadhana (Springer Publications), vol. 40(8), pp. 2429-2455. 
An Overview on Extension and Limitations of Macroscopic Darcy's Law for a Single and Multi-Phase Fluid Flow through a Porous Medium

[9] Diaz, C E., I Chatzis and F A L Dullien. (1987). "Simulation of capillary pressure curves using bond correlated site percolation on a simple cubic network". Transport in Porous Media, vol. 2., pp. 215-240.

[10] Ghassemi, A., and G. Suresh Kumar. (2007) "Changes in fracture aperture and fluid pressure due to thermal stress and silica dissolution/precipitation induced by heat extraction from subsurface rocks". Geothermics (Elsevier Science Publications), v. 36(2), pp. 115-140.

[11] Gray W G and K O’Neill. (1976). “On the Development of Dracy's law for the general equations for flow in Porous Media”. Water Resources Research, vol. 12(1)., pp. 148-154.

[12] Gray W G., and S M Hassanizadeh. (1989). "Averaging theorems and averaged equations for transport of interface properties in multi-phase systems". International Journal of Multi-Phase Flow, vol. 15(1)., pp. 81-95.

[13] Hassanizadeh M and W G Gray. (1990). "Mechanics and thermodynamics of multiphase flow in porous media including interface boundaries". Advances in Water Resources, vo. 13(4)., pp. 169-186.

[14] Jerauld G R., and S J Salter (1990). "The effect of pore-structure on hysteresis in relative permeability and capillary pressure: Pore level modelling”. Transport in Porous Media, vol., 5., pp. 103-151.

[15] Kalaydjian F. (1990). "Origin and quantification of coupling between relative permeabilities for two-phase flows in porous media”. Transport in Porous Media, vol. 5., pp. 215-229.

[16] Liu H., P R Patil., and U Narusawa. (2007). On Darcy-Brinkman Equation: Viscous flow between two parallel plates packed with regular square arrays of cyclinders. Entropy, vol. 9., pp. 118-131.

[17] Mohanasundaram. S., G. Suresh Kumar., and B. Narasimhan. (2013) "Numerical modeling of fluid flow through unsaturated zone using dual porosity approach". ISH Journal of Hydraulic Engineering (Taylor \& Francis), vol. 19, no. 2, pp. 97-110.

[18] Mohanasundaram S., BalajiNarasimhan., and G. Suresh Kumar. (2017). "Transfer Function Noise Modeling of Groundwater Level Fluctuation using Threshold Rainfall Based Binary Weighted Parameter Estimation Approach”. (DOI: http://dx.doi.org/10.1080/02626667.2016.1171325) Hydrological Sciences Journal (Taylor \& Francis Publications), vol. 62(1), pp. 36-49.

[19] Muccino J C., W G Gray., and L A Ferrand. (1998). “Toward an improved understanding of multiphase flow in porous media“. Reviews of Geophysics, vol. 36(3)., pp. 401-422.

[20] Natarajan, N., and G. Suresh Kumar. (2010) "Colloidal Transport in a Coupled Sinusoidal Fracture Matrix System”. International Journal of Geology (NAUN Publications), vol. 4(2), pp. 41-47.

[21] Natarajan, N., and G. Suresh Kumar. (2010) "Radionuclide and colloid co transport in a coupled fractureskin-matrix system”. Colloids and Surfaces A: Physicochemical and Engineering Aspects (Elsevier Publications), vol. 370(1-3), pp. 49-57

[22] Natarajan, N., and G. Suresh Kumar. (2011). "Numerical Modeling and Spatial Moment Analysis of Thermal Fronts in a Coupled Fracture-Skin-Matrix System”. Geotechnical and Geological Engineering (Springer Link Publications), vol. 29(4), pp. 477- 491.

[23] Natarajan. N., and G. Suresh Kumar. (2012a). "Effect of Fracture-Skin on Virus Transport in Fractured Porous Media". Geoscience Frontiers (Elsevier Publications), vol. 3(6), pp. 893-900. DOI: 10.1016/j.gsf.2012.03.004.

[24] Natarajan. N., and G. Suresh Kumar. (2012b). "Evolution of fracture permeability due to co-colloidal bacterial transport in a coupled fracture-skin-matrix system”. Geoscience Frontiers (Elsevier Publications), vol. 3(4), pp. 503-514.

[25] Natarajan, N., and G. Suresh Kumar. (2014a). "Effect of Fracture Skin in Clay Fractured Porous Media". ISH Journal of Hydraulic Engineering (Taylor \& Francis Publications), vol. 20(3), pp. 263-273.

[26] Natarajan. N., and G. Suresh Kumar. (2014b). "Lower order spatial moments for colloidal transport in a fracture-matrix coupled system". ISH Journal of Hydraulic Engineering (Taylor \& Francis Publications), vol. 20(2), pp. 200-211.

[27] Natarajan, N., and G. Suresh Kumar. (2015). Numerical Modeling and Spatial Moment Analysis of Solute Transport with Langmuir Sorption in a Fracture Matrix Coupled System. ISH Journal of Hydraulic Engineering (Taylor and Francis Publications), vol. 21(1)., pp. 28-41.

[28] Natarajan, N., and G. Suresh Kumar. (2016) "Effect of Sips Adsorption Isotherm on Contaminant Transport Mechanism in Fractured Porous Media “. KSCE Journal of Civil Engineering (Springer Publications), vol. 20(5)., pp. $1714-1720$.

[29] Natarajan, N., and G. Suresh Kumar. (2018). "Spatial Moment Analysis of Multispecies Contaminant Transport in Porous Media”. Environmental Engineering Research (KSEE Publishers), vol. 23(1)., pp. 76-83.

[30] Nikhil, B, L., and G. Suresh Kumar. (2015a). "Effect of Non-Linear Sorption on Multispecies Radionuclide Transport in Fracture-Matrix System with Variable Fracture Aperture: A Numerical Study". ISH Journal of Hydraulic Engineering (Taylor and Francis Publications), vol. 21(3), pp. 242-254. 
An Overview on Extension and Limitations of Macroscopic Darcy's Law for a Single and Multi-Phase Fluid Flow through a Porous Medium

[31] Nikhil, B, L., and G. Suresh Kumar. (2015b). "Thermal Front Propagation in Variable Aperture FractureMatrix System: A Numerical Study”. Sadhana (Springer Publications), vol. 40(2), pp. 605-622.

[32] Nikhil, B, L., and G. Suresh Kumar. (2016). "Numerical Modeling of Two Species Radionuclide Transport in a Single Fracture-Matrix System with Variable Facture Aperture". Geosciences Journal (Springer Publications), vol. 20(5)., pp. 627-638.

[33] Nikhil, B, L., and G. Suresh Kumar. (2017). "Effect of Random Fracture Aperture on the Transport of Colloids in a Coupled Fracture-Matrix System". Geosciences Journal (Springer Publications), vol. 21(1), pp. 55-69.

[34] Nitha N., G. Suresh Kumar., and I. M. Nambi. (2018). Analyzing the Flow of Energies Within the Well Capture Zones Under Steady State Conditions. (Avaialble Online). Groundwater for Sustainable Development (Elsevier Publications), vol. 6., pp. 134-140.

[35] OmkarGaonkar., G. Suresh Kumar., and I. M. Nambi. (2016a). "Numerical Investigations on Pesticide Fate and Transport in an Unsaturated Porous Medium for a Coupled Water and Pesticide Management". Environmental Earth Sciences (Springer Publications). Vol. 75:1232.

[36] OmkarGaonkar., G. Suresh Kumar., and I. M. Nambi. (2016b). "Numerical Modelling on Fate and Transport of Coupled Adsorption and Biodegradation of Pesticides in an Unsaturated Porous Medium". ISH Journal of Hydraulic Engineering (Taylor and Francis Publications), vol. 22(3)., pp. 236-246.

[37] Patwardhan, S. D., Fatemeh, F., Radhika, G., and G. Suresh Kumar. (2014) "Simulation and Mathematical Modeling of Stimulated Shale Gas Reservoirs" Industrial \& Engineering Chemistry Research (ACS Publications), vol. 53., pp. 19788-19805.

[38] Patwardhan, S., G. Radhika., and G. Suresh Kumar. (2017). Impact of Proppant Diagenesis on Shale Gas Productivity". International Journal of Oil, Gas and Coal Technology (Inderscience Publishers), vol. 14, No: 1(2)., pp. 147-171.

[39] Rakesh T V., and G. Suresh Kumar. (2016). "Numerical Modeling on the Sensitivity of Directional Dependent Interface Heat Transfer on Thermal Transport in a Coupled Fracture-Matrix System". Geosciences Journal (Springer Publications), vol. 20(5)., pp. 639-647.

[40] Renu, V. and Suresh Kumar, G. (2012). "Numerical Modeling and Spatial Moment Analysis of Solute Mobility and Spreading in a Coupled Fracture-Skin-Matrix System". Geotechnical and Geological Engineering (SpringerLink), vol. 30(6), pp. 1289-1302. DOI: 10.1007/s10706-012-9540-3.

[41] Renu, V., and G Suresh Kumar. (2014). "Temporal Moment Analysis of Solute Transport in a Coupled Fracture-Skin-Matrix System" Sadhana - Academy proceedings in Engineering Sciences (Springer Publications), vol. 39(2), pp. 487-509.

[42] Renu, V., and G Suresh Kumar. (2016a). "Temporal Moment Analysis of Multi-Species Radionuclide Transport in a Coupled Fracture-Skin-Matrix System with a Variable Fracture Aperture". Environmental Modeling and Assessment (Springer Publications), vol. 21(4)., pp. 547 - 562.

[43] Renu, V., and G Suresh Kumar. (2016b). "Numerical Modeling on Benzene Dissolution into Groundwater and Transport of Dissolved Benzene in a Saturated Fracture-Matrix System”. Environmental Processes, vol. 3(4)., pp. 781-802.

[44] Renu. V., and G. Suresh Kumar. (2017a). Multi-Component Transport of BTX in a Discretely Fractured Aquifer with Fracture-Skin: Numerical Investigation and Sensitivity analysis. (Ms. No. ENGE-D-1700102R1) Environmental Earth Sciences (Springer Link Publications) Vol. 76(17)., pp. 1-15.

[45] Renu V., and Suresh Kumar G. (2017b). Benzene Dissolution and Transport in a Saturated Sinusoidal Fracture with non-uniform Flow: Numerical Investigation and Sensitivity Analysis. Environmental Processes (SpringerLink Publications), vol. 4(3)., pp. 587-601.

[46] Renu V., and G. Suresh Kumar. (2018a). "Mathematical Modeling on Mobility and Spreading of BTEX in a Discretely Fractured Aquifer System under the Coupled Effect of Dissolution, Sorption, and Biodegradation”. Transport in Porous Media (Springer Publications), vol. 123(2), pp. 421-452.

[47] Renu, V., and G. Suresh Kumar. (2018b). "Interaction of Dissolution, Sorption and Biodegradation on Transport of BTEX in a Saturated Groundwater System: Numerical Modeling and Spatial Moment Analysis". Journal of Earth System Science (Springer Publications), vol. 127(4), pp. 1-21,

[48] Rockey Abhishek., G. Suresh Kumar., and R. K. Sapru. (2015). "Wettability Alteration in Carbonate Reservoirs using Nano-Fluids". Petroleum Science and Technology (Taylor and Francis Publications), vol. 33(7), pp. 794-801.

[49] Rockey Abhishek., B. L. Nikhil., and G. Suresh Kumar. (2016). "Effect of Transverse Forces on Velocity of Nano-particles through a Single Facture in a Fractured Petroleum Reservoir". International Journal of Oil, Gas and Coal Technology (Inderscience Publishers), vol. 12(4)., pp. 359-378. 
An Overview on Extension and Limitations of Macroscopic Darcy's Law for a Single and Multi-Phase Fluid Flow through a Porous Medium

[50] Samarth D. Patwardhan., N. Bhore., A. Banerjee., and G. Suresh Kumar. (2015). "Impact of dynamic slippage on productivity of shale reservoirs", vol. 12(5), pp. 443-451. DOI:10.1260/1708-5284.12.5.443 World Journal of Engineering (ISSN: 1708-5284).

[51] Samarth Patwardhan., FatemehFamoori., and G. Suresh Kumar. (2016). "Quad Porosity Shale Systems A Review”. World Journal of Engineering (Multi-Science Publications), vol. 13(6). 2016 Dec5.

[52] Samarth D. Patwardhan., Deepak Singh., Rockey Abhishek., G. Suresh Kumar. (2017). "Modelling of mineral precipitation in fractures with variable aperture". ISH Journal of Hydraulic Engineering (Taylor \& Francis Publications), Vol. 23(2)., pp. 203-211.

[53] Siddiqui F., M Y Soliman., W House., and AIbragimov. (2015). Pre-Darcy flow revisited under experimental investigation. Journal of Petroleum \& Environmental Biotechnology, vol. 6(6)., pp. 1-5. DOI: $10.4172 / 2157-7463.1000249$

[54] Sivasankar, P., and G. Suresh Kumar. (2014). Numerical Modeling of Enhanced Oil Recovery by Microbial Flooding under Non-isothermal Conditions. Journal of Petroleum Science and Engineering (Elsevier Publications), vol. 124., pp. 161-172.

[55] Sivasankar P., Rajesh Kanna., G Suresh Kumar., and S N Gummadi. (2016) "Numerical Modelling of Biophysicochemical effects on Multispecies Reactive Transport in Porous Media Involving Pseudomonas Putida for Potential Microbial Enhanced Oil Recovery Application”. Bioresource Technology (Elsevier Publications), vol. 211., pp. 348 - 359.

[56] Sivasankar P., and G. Suresh Kumar. (2017a). Improved Empirical Relations for Estimating Original Oil in Place Recovered During Microbial Enhanced Oil Recovery Under Varied Salinity Conditions. Petroleum Science and Technology (Taylor \& Francis Publications), vol. 35(21)., pp. 2036-2043.

[57] Sivasankar, P., and G. Suresh Kumar. (2017b). "Influence of pH on dynamics of Microbial Enhanced Oil Recovery Processes using Biosurfactant Produced Pseudomonas Putida: Mathematical Modeling and Numerical Simulation”. Bioresource Technology (Elsevier Publications), vol. 224., pp. 498-508.

[58] Sivasankar P., and G. Suresh Kumar. (2018). "Modelling the influence of interaction between injection and formation brine salinities on in-situ microbial enhanced oil recovery processes by coupling of multiple-ion exchange transport model with multiphase fluid flow and multi-species reactive transport models". Journal of Petroleum Science and Engineering (Elsevier Publications), vol. 163, pp. 435-452.

[59] Sivasankar P., and G. Suresh Kumar. (2019). "Influence of Bio-Clogging Induced Formation Damage on Performance of Microbial Enhanced Oil Recovery Processes”. Fuel (Elsevier Publications), vol. 236, pp. 100-109.

[60] Srinivasa Reddy and G. Suresh Kumar. (2014). A comprehensive analysis on thermal and kinetic aspects of in-situ combustion: Numerical Approach. Applied Mechanics and Materials, vol. 592-594, pp. 13931397.

[61] Srinivasa Reddy, D., and G. Suresh Kumar. (2015a). A Numerical Study on Phase Behavior effects in Enhanced Oil Recovery by In-Situ Combustion. Petroleum Science and Technology (Taylor and Francis Publications), vol. 33(3)., pp. 353-362.

[62] Srinivasa Reddy and G. Suresh Kumar. (2015b). "Numerical Simulation of Heavy Crude Oil Combustion in Porous Combustion-Tube”. Combustion Science \& Technology (Taylor \& Francis Publications), vol. 187(12), pp. 1905-1921.

[63] Suresh Kumar. G. (2009) “Influence of Sorption Intensity on Solute Mobility in a Fractured Formation”. Journal of Environmental Engineering (ASCE), 135(1), pp. 1-7.

[64] Suresh Kumar, G., M. Sekhar and D Mishra. (2008) "Time dependent dispersivity of linearly sorbing solutes in a single fracture with matrix diffusion”. Journal of Hydrologic Engineering (ASCE), 13(4), pp. 250-257.

[65] Suresh Kumar. G. (2008) "Effect of Sorption Intensities on Dispersivity and Macro-dispersion Coefficient in a Single Fracture with Matrix Diffusion”. Hydrogeology Journal (Springer), 16(2), pp. 235-249.

[66] Sekhar M., G. Suresh Kumar and D Mishra. (2006) "Numerical Modeling and Analysis of Solute Velocity and Macrodispersion for Linearly and Nonlinearly Sorbing Solutes in a Single Fracture with Matrix Diffusion". Journal of Hydrologic Engineering (ASCE), v. 11(4), pp. 319-328.

[67] Suresh Kumar G., and A. Ghassemi. (2006) "Spatial Moment Analysis for One-Dimensional Nonisothermal Quartz Transport and Dissolution/Precipitation in a Fracture-Matrix System". Journal of Hydrologic Engineering (ASCE), v. 11(4), pp. 338-346.

[68] Sekhar M., and G. Suresh Kumar. (2006) "Modeling Transport of Linearly Sorbing Solutes in a Single Fracture: Asymptotic Behavior of Solute Velocity and Dispersivity". Geotechnical and Geological Engineering (Springer Science Publications) - Vol. 24(1), pp. 183-201. 
An Overview on Extension and Limitations of Macroscopic Darcy's Law for a Single and Multi-Phase Fluid Flow through a Porous Medium

[69] Suresh Kumar. G., M. Sekhar., and D. Misra (2006) "Time Dependent DispersivityBehavior of NonReactive Solutes in a System of Parallel Fractures”. Hydrology and Earth System Sciences Discussions (Copernicus Publications) - vol. 3(3), pp. 895-923.

[70] Suresh Kumar G., and M. Sekhar. (2005) "Spatial Moment Analysis for Transport of Nonreactive Solutes in a Fracture-Matrix System”. Journal of Hydrologic Engineering (ASCE), v. 10(3), pp. 192-199.

[71] Suresh Kumar. G., and A. Ghassemi. (2005) "Numerical Modeling of Non-Isothermal Quartz Dissolution/Precipitation in a Coupled Fracture-Matrix System". Geothermics (Elsevier Science Publications), v. 34(4), pp. 411-439.

[72] Suresh Kumar. G. (2014a). "Mathematical Modeling on Transport of Petroleum Hydrocarbons in Saturated Fractured Rocks" Sadhana - Academy proceedings in Engineering Sciences (Springer Publications), vol. 39(5)., pp. 1119-1139.

[73] Suresh Kumar, G. (2014b). "Mathematical Modeling of Groundwater Flow and Solute Transport in a Saturated Fractured Rock using Dual-Porosity Approach". Journal of Hydrologic Engineering (ASCE), vol. 19(12)., pp. 04014033-1 - 04014033-8.

[74] Suresh Kumar G and T V Rakesh. (2015). "Numerical Modeling of Reactive Solute Transport in a Single Fracture with Matrix Diffusion under Complex Boundary Condition". ISH Journal of Hydraulic Engineering (Taylor and Francis Publications), vol. 21(2), pp. 125-141.

[75] Suresh Kumar, G. (2015). "Subsurface Transport of Nuclear Wastes in the Indian Subcontinent". ISH Journal of Hydraulic Engineering (Taylor and Francis Publications), vol. 21(2), pp. 162-176.

[76] Suresh Kumar G. (2016). "Modeling Fluid Flow through Fractured Reservoirs: Is it different from Conventional Classical Porous Medium?". Current Science (Current Science Association \& Indian Academy of Sciences), vol. 110(4)., pp. 695-701.

[77] Suresh Kumar G., and D. Srinivasa Reddy. (2017). "Numerical Modeling of Forward In-Situ Combustion Process in Heavy Oil Reservoirs". International Journal of Oil, Gas and Coal Technology (Inderscience Publishers), Vol. 16(1)., pp. 43-58.

[78] Suresh Kumar G., and T. V. Rakesh. (2018). "Numerical Modeling of Hyperbolic Dominant Transient Fluid Flow in Saturated Fractured Rocks using Darcian Approach". Groundwater for Sustainable Development (Elsevier Publications), vol. 7., pp. 56-72.

[79] TapanKidambi., and G. Suresh Kumar. (2016). "Mechanical Earth Modeling for a Vertical Well Drilled in a Naturally Fractured Tight Carbonate Reservoir in the Persian Gulf”. Journal of Petroleum Science and Engineering (Elsevier Publications), vol. 141., pp. 38-51.

[80] TapanKidambi.,AwezHanegaonkar., Ankit Dutt., and G. Suresh Kumar. (2017). “A Fully Coupled Flow and Geomechanics Model for a Tight Gas Reservoir: Implications for Compaction, Subsidence and Faulting". Journal of Natural Gas Science and Engineering (Elsevier Publications), vol. 38, pp. 257-271.

[81] Teng H., and T S Zhao. (2000). An extension of Darcy's law to non-Stokes flow in porous media. Chemical Engineering Science, vol. 55., pp. 2727-2735.

[82] Tushar Sharma., G. Suresh Kumar., and JitendraSangwai. (2014a). "Viscosity of the Oil-in-Water Pickering Emulsion Stabilized by Surfactant-Polymer and Nanoparticle-Surfactant-Polymer System”. Korea-Australia Rheology Journal (Springer Publications), vol. 26(4)., pp. 1-11.

[83] Tushar Sharma., G. Suresh Kumar., and JitendraSangwai. (2014b). "Enhanced Oil Recovery using Oil-inWater (O/W) Emulsion Stabilized by Nanoparticle, Surfactant and Polymer in the Presence of $\mathrm{NaCl}^{\prime}$. Geosystem Engineering (Taylor \& Francis Publications), vol. 17(3)., pp. 195-205.

[84] Tushar Sharma., G. Suresh Kumar., and JitendraSangwai. (2015a). "Viscoelastic Properties of Oil-inWater (O/W) Pickering Emulsion Stabilized by Surfactant-Polymer and Nanoparticle-Surfactant-Polymer System" Industrial \& Engineering Chemistry Research (ACS Publications), vol. 54(5), pp. 1576-1584.

[85] Tushar Sharma., G. Suresh Kumar., Bo Hyun Chon., and JitendraSangwai. (2015b). "Thermal Stability of Oil-in-Water Pickering Emulsion in the Presence of Nanoparticle, Surfactant and Polymer" Journal of Industrial and Engineering Chemistry (Elsevier Publications), vol. 22, pp. 324-334.

[86] Tushar Sharma., G. Suresh Kumar., and JitendraSangwai. (2015c). Comparative Effectiveness of Production Performance of Pickering Emulsion Stabilized by Nanoparticle-Surfactant-Polymer over Surfactant-Polymer (SP) Flooding for Enhanced Oil Recovery for Brownfield Reservoir" Journal of Petroleum Science and Engineering (Elsevier Publications), vol. 129, pp. 221-232.

[87] Vasudevan, M., G. Suresh Kumar., and I. M. Nambi. (2014). "Numerical Study on Kinetic/Equilibrium Behavior of Dissolution of Toluene under Variable Sub-Surface Conditions". European Journal of Environmental and Civil Engineering (Taylor \& Francis Publications), vol. 18(9), pp. 1070-1093. 
An Overview on Extension and Limitations of Macroscopic Darcy's Law for a Single and Multi-Phase Fluid Flow through a Porous Medium

[88] Vasudevan, M., G. Suresh Kumar., and I. M. Nambi. (2014). "Numerical Modeling of Multi-Component LNAPL Dissolution Kinetics at Residual Saturation in a Saturated Sub-Surface System". Sadhana Academy proceedings in Engineering Sciences (Springer Publications), vol. 39(6)., pp.1387-1408.

[89] Vasudevan, M., G. Suresh Kumar., and I. M. Nambi. (2015). "Numerical Modeling on Kinetics of Sorption and Dissolution and their interactions for Estimating Mass Removal of Toluene from Entrapped Soil Pores”. Arabian Journal of Geosciences (Springer Publications), vol. 8(9), pp. 6895-6910.

[90] Vasudevan., I M Nambi., and G. Suresh Kumar. (2016a) "Scenario-based Modeling of Mass Transfer Mechanisms at a Petroleum Contaminated Field Site - Numerical Implications". Journal of Environmental Management (Elsevier Publishers), vol. 175., pp. 9-19.

[91] Vasudevan. M., G. Suresh Kumar., and I. M. Nambi. (2016b). "Numerical Modeling on Rate Limited Dissolution Mass Transfer of Entrapped Petroleum Hydrocarbons in a Saturated Sub-Surface System". ISH Journal of Hydraulic Engineering (Taylor and Francis Publications), vol. 22(1), pp. 3-15.

[92] Vasudevan, M., C.D. Johnston, T.P. Bastow, G. Lekmine, J.L. Rayner, I.M. Nambi, G. Suresh Kumar, R. Ravi Krishna, G.B. Davis. (2016c). "Effect of compositional heterogeneity on dissolution of non-ideal LNAPL mixtures”. Journal of Contaminant Hydrology (Elsevier Publications). vol. 194., pp. 10-16.

[93] Vasudevan M., M. Berlin., S. Mohanasundaram., G. Suresh Kumar., and I. M. Nambi. (2017). Numerical Investigations on Feasibility of Surfactant Enhanced Remediation of Polycyclic Aromatic Hydrocarbon in an Unsaturated Subsurface System beneath an Onshore Surface Spill Site. International Journal of Environmental Technology and Management (Inderscience Publications), vol. 20, no. 5/6, pp. 321-346.

[94] Vivek R., and G. Suresh Kumar. (2016). "Numerical Investigation on Effect of Varying Injection Scenario and Relative Permeability Hysteresis on CO2 Dissolution in Saline Aquifer". Environmental Earth Sciences (Springer Publications). Vol. 75:1192.

[95] Vivek, R., P. Sivasankar., and G. Suresh Kumar. (2017). Accelerating Dissolution Trapping by Low Saline WAG Injection Scenario. Energy Procedia (Elsevier Publications), vol. 114, pp. 5038-5047.

[96] Whitaker S. (1986). Flow in porous media I: A theoretical derivation of Darcy's law. Transport in porous media, vol. 1., pp. 3-25.

Citation: Suresh Kumar Govindarajan, (2019)" An Overview on Extension and Limitations of Macroscopic Darcy's Law for a Single and Multi-Phase Fluid Flow through a Porous Medium", Southeast Cameroon, International Journal of Mining Science (IJMS), 5(4), pp.1-21, DOI: http://dx.doi.org/10.20431/24549460.0504001

Copyright: () 2019 Authors. This is an open-access article distributed under the terms of the Creative Commons Attribution License, which permits unrestricted use, distribution, and reproduction in any medium, provided the original author and source are credited 